\title{
Demographic Undertones for Sepsis Mortality in a Community-Based Hospital
}

\author{
Ahmad Jabri ${ }^{\mathrm{a}, \mathrm{g}}$, Cosmo Fowler ${ }^{\mathrm{b}}$, Yashu Dhamija ${ }^{\mathrm{a}}$, Jafar Alzubi ${ }^{\mathrm{a}}$, Smriti Bhatia ${ }^{\mathrm{c}}$, \\ Ahmad Al-abdouh ${ }^{\mathrm{d}}$, Anas Alameh ${ }^{\mathrm{a}}$, Hamzeh Alfahel ${ }^{\mathrm{a}}$, Faris Haddadin ${ }^{\mathrm{b}}$, \\ Zaid Shahrorie, Farhan Nasser ${ }^{\mathrm{f}}$, Ahmad Ababneh ${ }^{\mathrm{a}}$
}

\begin{abstract}
Background: Sepsis continues to take main stage in healthcare. Therefore, it remains crucial to elucidate contributors to sepsis mortality. The aim of this study is to determine the impact of race, insurance type, and code status on sepsis mortality in a community health system.
\end{abstract}

Methods: We conducted a retrospective cohort study of inpatient adults of any sex, race, and insurance type with a diagnosis of sepsis, severe sepsis, septic shock, or pneumonia.

Results: We included 913 patients, with an average age of 69 years for expired patients and 62 years for non-expiring patients $(\mathrm{P}<$ $0.0001)$. After controlling for other variables, patients who presented as comfort care arrest were 4.3 (95\% confidence interval (CI): 1.8 to $9.9, \mathrm{P}=0.0007)$ times more likely to have died than full code patients. Those who were comfort care only were 10.6 (95\% CI: 0.8 to 140.6 , P $=0.0741$ ) times more likely to have died than the full code, although this was not statistically significant.

Conclusions: The results suggest that patients who are comfort care arrest have an increased risk of sepsis mortality. The results show no impact of insurance type or race on sepsis mortality, which is in contrast to some existing literature. The study suggests that institutions may need to investigate internal variables related to sepsis mortality.

Keywords: Sepsis; Mortality; Race; Code status; Insurance; Community health

Manuscript submitted October 17, 2021, accepted November 2, 2021

Published online January 29, 2022

aDepartment of Internal Medicine, Cleveland Clinic Akron General, Akron, OH, USA

${ }^{b}$ Department Cardiology, Baylor College of Medicine, Houston, TX, USA

'Department of Internal Medicine, Nazareth Hospital, Philadelphia, PA, USA

${ }^{\mathrm{d}}$ Department of Medicine, Saint Agnes Hospital, Baltimore, MD, USA

eDepartment of Internal Medicine, Hashemite University, Amman, Jordan

${ }^{\mathrm{f}} \mathrm{Heart}$ and Vascular Center, Case Western Reserve University/MetroHealth Medical Center, Cleveland, OH, USA

gCorresponding Author: Ahmad Jabri, Heart and Vascular Center, MetroHealth Medical Center, Cleveland, OH 44109, USA. Email: ajabri@metrohealth.org

doi: https://doi.org/10.14740/jocmr4618

\section{Introduction}

Sepsis is the body's overwhelming and life-threatening inflammatory response to an infection that can result in tissue damage, organ dysfunction, and death [1]. Mortality resulting from sepsis can exceed $10 \%$ and reach more than $40 \%$ in septic shock cases [1]. Sepsis mortality can be influenced by multiple factors ranging from patient-related to hospital-related problems. Multiple studies have been carried out to assess the factors associated with high mortality and methods of amending them for better survival.

The effects of ethnicity, insurance, and code status at presentation on sepsis mortality have been studied, but the results are inconclusive [2]. For example, one large study reported that the rate of African American and Hispanic patient deaths related to sepsis was higher than sepsis-related deaths in Caucasian patients [3]. However, other studies found that African American patients had lower sepsis mortality than Caucasian patients [2, $4,5]$. On the other hand, another study found no association between ethnicity and sepsis mortality [6]. Concerning insurance status, one study found that patients with Medicaid and private insurance had higher sepsis-related mortality compared to patients with Medicare [2, 7]. Additionally, uninsured patients had an even higher mortality rate when compared to other insurance types $[2,7]$. It has been argued that the patients with Medicaid have underlying diseases for which they are treated, and a subsequent infection may be more challenging to treat. Moreover, it is believed that uninsured patients tend to delay seeking medical advice until complications arise [7]. Finally, studies on the impact of specified code status, do not resuscitate-arrest (DNRA) and do not resuscitate-comfort care only (DNR-CCO) versus full code, indicate that patients with a prior DNR or DNR-CCO code status carry a higher mortality rate as it appears to limit the management and care of sepsis patients.

At our institution, we have observed a significant increase in sepsis-related mortality over the last 2 years. As such, the objective of this study will be to investigate the effect of ethnicity, insurance type, and code status on sepsis-related mortality during the 2-year period at our institution. Due to inconsistencies in the literature regarding the reported impact of these factors on sepsis-related mortality to date, information from this study will add to the current literature, and hopefully, shed light on whether these factors significantly impact the mortality associated with sepsis at our institution and more broadly. 


\section{Materials and Methods}

\section{Data sources}

The study procedures were approved by our internal research review board. Consent was waived by our review board. This study was conducted with the ethical standard of the responsible institution on human subjects as well as with the Helsinki Declaration.

Analysis used a chart review of admitted patients at Cleveland Clinic Akron General in Akron, OH, between March 30, 2016, and June 30, 2017. The information was obtained from scanned written medical records of patients. Our institution transitioned to a fully electronic medical record in October 2017.

Study data were collected and managed using Research Electronic Data Capture (REDCap) hosted at Cleveland Clinic $[8,9]$. REDCap is a secure, web-based software platform designed to support data capture for research studies, providing: 1) an intuitive interface for validated data capture; 2) audit trails for tracking data manipulation and export procedures; 3) automated export procedures for seamless data downloads to common statistical packages; 4) procedures for data integration and interoperability with external sources.

This study was a retrospective cohort review of patients admitted with diagnoses of sepsis, severe sepsis, septic shock, and/or pneumonia between March 30, 2016, and June 30, 2017. Patients 18 years of age and older, of any sex or race, and any insurance with infection or suspected infection on admission will be eligible for inclusion. Patients will be excluded if they are under 18 years of age, have an underlying disease that would decrease their life expectancy. The patients' records were reviewed for the initial diagnosis of sepsis using the International Classification of Disease (ICD) (ICD Eleventh Revision (ICD-11)) coding and only patients with severe sepsis and septic shock will be included.

\section{Outcomes}

The primary outcome of this study was mortality. Measures in this study included race, insurance type, and code status. Code status was measured at the time of admission, discharge, and, if applicable, time of death. If the patient's code status was modified, mortality was linked to the latest change in code status. Clinical information such as presenting history, lactate, and blood cultures were used to confirm the diagnoses of sepsis. Additional comorbidities were measured by clinical diagnosis or disease-specific testing.

\section{Statistical analyses}

Categorical variables are reported using frequencies and percentages, and $\mathrm{P}$ values obtained from the Chi-square test or the Fisher's exact test where appropriate. $T$-tests were used to compare continuous data across groups and reported as mean and standard deviation (SD). Variables that are with highly skewed distributions were log-transformed before analyzing the data. Univariable logistic regression analysis was used to calculate odds ratios associating individual predictors of interest and mortality. Adjusted odds ratios (aORs) were calculated using multiple logistic regression analysis. For continuous variables, we express the odds ratio per 1-SD increase in the variable (or its log-transformed value). A significance level of 0.05 was assumed for all tests. Analysis was done using SAS ${ }^{\circledR}$ Software (version 9.4; Cary, NC).

\section{Results}

There were 917 records in the REDCap project. Four records were excluded from analysis, two of which were duplicates and two were missing outcome variable data. Patients with multiple admissions were included for analysis. Tables 1 and 2 describe the baseline characteristics and demographics of patients included in the study. The mean age of all patients was 62.7 , with the average age for patients who expired significantly greater than those who survived (69.3 vs. $61.9, \mathrm{P}<$ $0.0001)$. Female patients were $47 \%(50 / 106)$ of expired and $52 \%$ (417/807) of surviving patients. Overall, 83.0\% (749/903) of the patients in the study were White, $16.4 \%(148 / 903)$ were Black, and $0.7 \%(6 / 903)$ were of other races. Of all included patients, 49.4\% (450/911) had Medicare, 22.4\% (204/911) had Medicaid, 26.2\% (239/911) were privately insured, and $2 \%$ (18/911) were self-pay. At admission, 89.3\% (814/912) of patients were full code, $5 \%$ being DNR-comfort care arrest (CCA)/do not intubate (DNI) (45/912), and 5.5\% (50/912) being DNR-CCA. The transition of code status happened in $27 \%$ (244/904) of patients during the reviewed admission.

Tables 1 and 2 show the results of bivariate analyses, comparing each variable with mortality without controlling for other variables. Based on these two tables, the results show that code status, both on admission and discharge, significantly affected hospital mortality. Those with DNR-CCO had the highest mortality rate $(33 \%, 1 / 3)$, followed by DNR-CCA $(30 \%, 15 / 50)$. Additionally, those with code status change during their hospital stay had a higher mortality rate than those who did not have code status change, 35\% (85/105) versus 3\% (20/105). Insurance type and ethnicity did not significantly impact the mortality rate $(\mathrm{P}=0.1234$ and $\mathrm{P}=0.6630$, respectively).

Table 3 shows the results of a multiple logistic regression assessing the effect code status on admission, insurance status, and ethnicity on hospital mortality after controlling for other pertinent variables. After controlling for other pertinent variables, DNR-CCO patients were 10.57 times $(0.80-140.55, \mathrm{P}$ $=0.0741)$ more likely to have died than those on full code, although this was not statistically significant. Those who were DNR-CCA were 4.26 times (1.84 - 9.86, $\mathrm{P}=0.0007)$ more likely to have died than those on full code. Those who had code status change were 12 times $(6.86-21.33, \mathrm{P}<0.0001)$ more likely to have died than those who had no code status change.

There were no significant differences in mortality when comparing Black patients to White patients (aOR: 1.59, confidence interval (CI): $0.80-3.15, \mathrm{P}=0.1866$ ). Additionally, there was no significant difference in mortality outcomes 
Table 1. Demographics and Other Characteristics of the Participants $(N=913)$

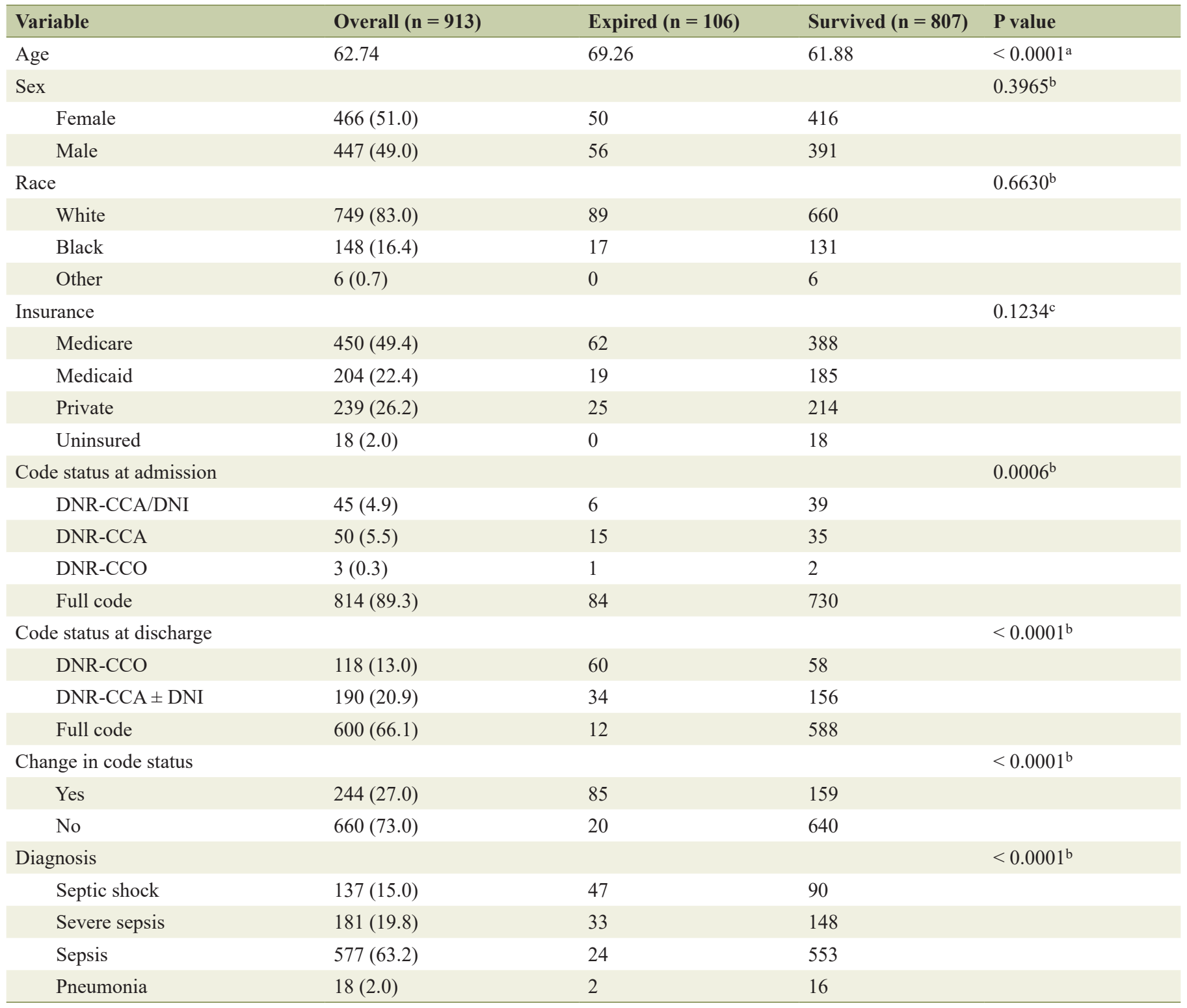

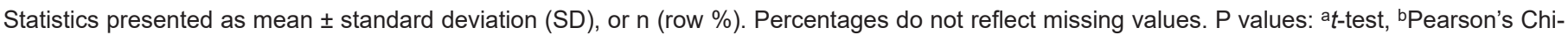
square test, 'Fisher's exact test. DNR: do not resuscitate; CCA: comfort care arrest; CCO: comfort care only; DNI: do not intubate.

of White patients to patients of other races (aOR: 1.65, CI: 0.05 - 56.34, $\mathrm{P}=0.7808)$. Additionally, age has no significant impact on mortality outcomes (aOR: 1.00, CI: $0.98-1.02, \mathrm{P}$ $=0.6861)$. Finally, there was no significant impact of insurance type on mortality when comparing Medicaid to Medicare (aOR: $1.22, \mathrm{CI}: 0.56-2.66, \mathrm{P}=0.6125$ ), private insurance to Medicare (aOR: 1.10, CI: $0.60-2.01, \mathrm{P}=0.7621$ ), or self-pay to Medicare (aOR: 0.77, CI: $0.04-15.75, \mathrm{P}=0.8658$ ).

\section{Discussion}

In this analysis of 913 patients diagnosed with sepsis and/or pneumonia, race and insurance did not significantly increase the mortality risk. The majority of patients in this study were Medicare or privately insured, followed by Medicaid and then self-pay. A majority of the patients were White, with the remainder being predominantly Black. This suggests that disparities recognized in race and insurance may have narrowed for sepsis mortality outcomes within our system. Our results reinforce a relationship between age and sepsis mortality, with the average age of patients who expired being 69 compared to 62 for those who did not expire $(\mathrm{P}<0.0001)$.

However, patients who were CCA at the time of admission were at a significantly increased risk of sepsis mortality (aOR: 4.3, CI: 1.8 to $9.9, \mathrm{P}=0.0007)$. While there is a trend for $\mathrm{CCO}$ 
Table 2. Comorbidities of Participants $(N=913)$

\begin{tabular}{lllll}
\hline Variable & Overall $(\mathbf{n = 9 1 3 )}$ & Expired $(\mathbf{n = 1 0 6})$ & Survived $(\mathbf{n}=\mathbf{8 0 7})$ & P value \\
\hline Arrhythmia & $204(22.3)$ & $28(13.7)$ & $176(86.3)$ & $0.2845^{\mathrm{b}}$ \\
Coronary artery disease & $207(22.7)$ & $35(16.9)$ & $172(83.1)$ & $103(81.1)$ \\
Cancer & $127(13.9)$ & $24(18.9)$ & $138(83.1)$ & $0.0068^{\mathrm{b}}$ \\
Congestive heart failure & $166(18.2)$ & $28(16.9)$ & $293(90.2)$ & $0.0057^{\mathrm{b}}$ \\
Diabetes & $325(35.6)$ & $32(9.9)$ & $234(84.8)$ & $0.2161^{\mathrm{b}}$ \\
Hyperlipidemia & $276(30.2)$ & $42(15.2)$ & $398(86.5)$ & $0.0251^{\mathrm{b}}$ \\
Hypertension & $460(50.4)$ & $62(13.5)$ & $48(82.8)$ & $0.0758^{\mathrm{b}}$ \\
Immunocompromised & $58(6.4)$ & $10(17.2)$ & $27(96.4)$ & $0.1665^{\mathrm{b}}$ \\
IV drug use & $28(3.1)$ & $1(3.6)$ & $47(78.3)$ & $0.2385^{\mathrm{c}}$ \\
Chronic lung disease & $60(6.6)$ & $13(21.7)$ & $203(92.7)$ & $0.0119^{\mathrm{b}}$ \\
Obesity & $219(24.0)$ & $16(7.3)$ & $81(87.1)$ & $0.0226^{\mathrm{b}}$ \\
Chronic respiratory failure & $93(10.2)$ & $12(12.9)$ & $42(82.4)$ \\
Valvular heart disease & $51(5.6)$ & $9(17.7)$ & $0.6812^{\mathrm{b}}$ & $0.1660^{\mathrm{b}}$ \\
\hline
\end{tabular}

Statistics presented as n (row \%). Percentages do not reflect missing values. P values: at-test, bPearson's Chi-square test, ' Fisher's exact test.

patients to be at increased risk, the results were not significant (aOR: 10.6, CI: 0.8 to $140.6, \mathrm{P}=0.1136$ ). The absolute number of patients who presented as $\mathrm{CCO}$ was low at 3 , showing an underrepresentation of these patients in the study. Regardless, it would follow that $\mathrm{CCO}$ patients would not receive care targeted towards reducing mortality.

Patients who are CCA would not be expected to have a change in treatment for sepsis compared to full code patients, except during the event of cardiopulmonary arrest. Generally, patients with multiple comorbidities and poor prognoses are targeted for goals of care discussions. Our study was not designed to investigate the comorbidities associated among code status subtypes, so we cannot comment on the underlying reasons for these patients being CCA at the time of admission. However, this hypothesis could explain why CCA patients were 4.3 times more likely to expire due to sepsis or pneumo-

Table 3. Multiple Logistic Regression Estimates of the Effect of Code Status, Insurance, and Ethnicity on Hospital Mortality (The Adjusted Factors Are Age, Code Status, Diagnosis, and Gender)

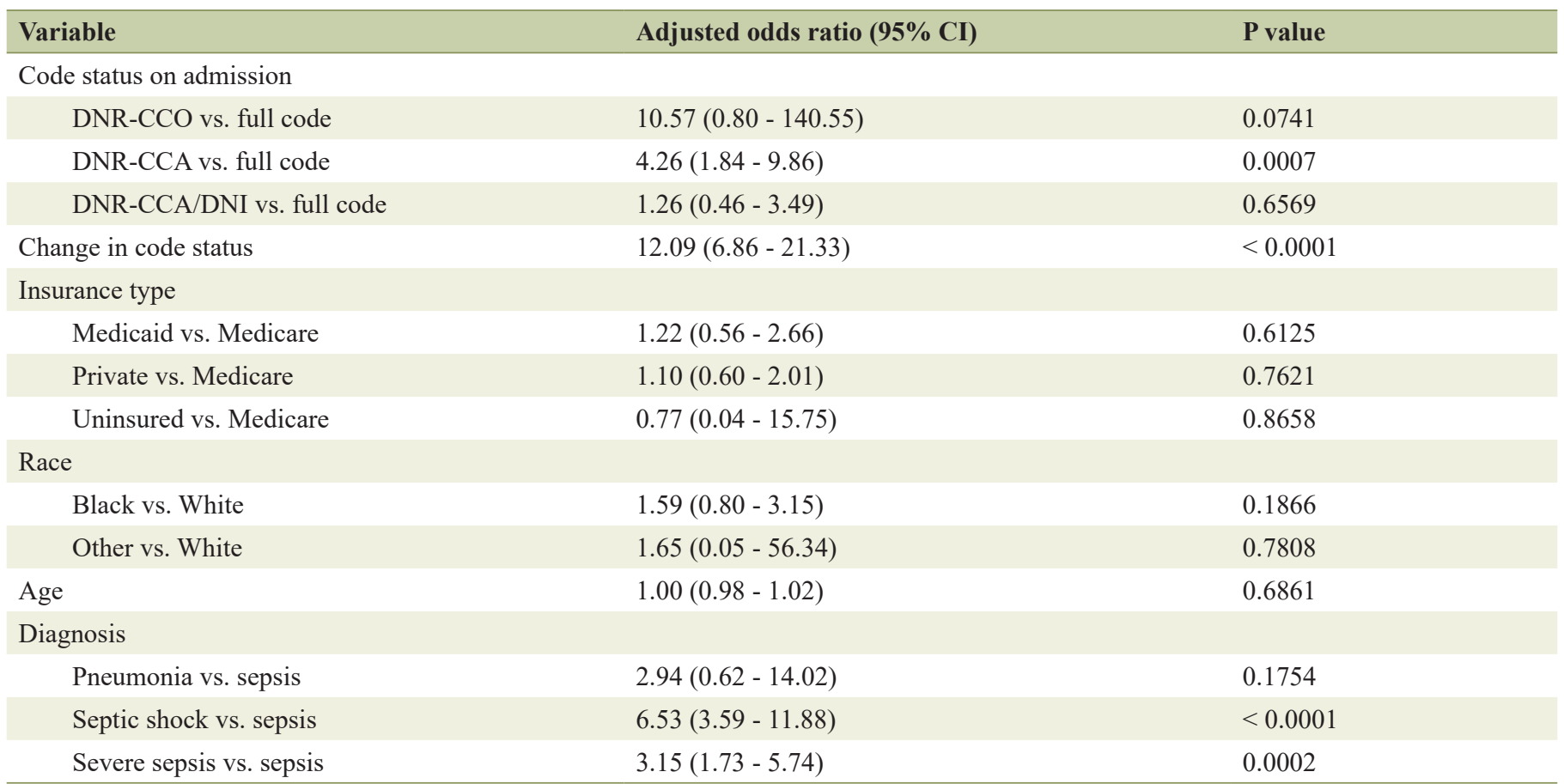

CI: confidence intervals; DNR: do not resuscitate; CCA: comfort care arrest; CCO: comfort care only; DNI: do not intubate. 
nia. Patients who had a code status change during their hospital stay had a higher mortality rate than those who did not have code status change.

This study was not designed to determine whether a code status change was from full code to DNR or vice versa. Despite the relationship between a change of code status and mortality rate, a greater percentage of patients with code status changes survived their infection $(65.2 \%)$ compared to those who expired $(34.8 \%, \mathrm{P}<0.0001)$. We believe this indicates there may not have been an association between the provider's interpretation of prognosis and changes in code status. Moreover, there may have been changes in code status leading to patients being discharged to another facility or home, where they may have expired. This outcome would not have been represented in our review of paper charts.

\section{Limitations}

While the study investigated the effects of insurance and race on sepsis mortality, it was not designed to identify confounding factors outside of age, gender, and comorbidities. Differences such as the units' level of care the patient was admitted to, primary service specialty or adherence to sepsis bundles were not measured. These are variables that could undermine our results. As alluded to above, our study was not designed to describe the events leading to code status changes.

A major limitation for evaluating mortality in this patient population is using written records that are retrospectively added to an electronic record management system. This disallows the evaluation of mortality in patients who may be discharged to a skilled nursing facility or home and expired within 30 days without acknowledgment within the paper chart. Regarding insurance type, the study was not designed to analyze the granularity of insurers for Medicaid and Medicare. Additionally, as the study focuses on demographics and sepsis mortality, it was not designed to evaluate variables such as zip codes and socioeconomic status that may further delineate risks for sepsis mortality.

\section{Conclusions}

Among 876 patients diagnosed with pneumonia, sepsis, severe sepsis, and septic shock, those admitted with a DNR-CCA code status were at significantly increased risk of mortality. In contrast, insurance and race had no significant impact on sepsis mortality.

\section{Acknowledgments}

None to declare.

\section{Financial Disclosure}

There was no specific funding source to be mentioned.

\section{Conflict of Interest}

The authors declare that they have no conflict of interest.

\section{Informed Consent}

The study procedures were approved by our internal research review board. Consent was waived by our review board.

\section{Author Contributions}

Dr. A. Jabri had full access to all of the data in the study and takes responsibility for the integrity of the data and the accuracy of the data analysis. Concept and design: A. Jabri, C. Fowler, Y. Dhamija, A. Al-abdouh and A. Ababneh. Acquisition, analysis, or interpretation of data: A. Jabri, Y. Dhamija, J. Alzubi, H. Alfahel and A. Ababneh. Drafting of the manuscript: A. Jabri, C. Fowler, Y. Dhamija, H. Alfahel, F. Haddadin, A. Al-abdouh, Z. Shahrori and F. Nasser. Critical revision of the manuscript for important intellectual content: A. Jabri, C. Fowler, Y. Dhamija, J. Alzubi, S. Bhaita, H. Alfahel, F. Haddadin, Z. Shahrori, A. Al-abdouh, F. Nasser and A. Ababneh. Statistical analysis: A. Jabri, H. Alfahel and A. Ababneh. Administrative, technical, or material support: A. Jabri, A. Alameh, H. Alfahel, A. Al-abdouh and F. Haddadin. Supervision: A. Jabri, A. Ababneh and Y. Dhamija.

\section{Data Availability}

Any inquiries regarding supporting data availability of this study should be directed to the corresponding author.

\section{References}

1. Singer M, Deutschman CS, Seymour CW, Shankar-Hari M, Annane D, Bauer M, Bellomo R, et al. The third international consensus definitions for sepsis and septic shock (Sepsis-3). JAMA. 2016;315(8):801-810.

2. Banta JE, Joshi KP, Beeson L, Nguyen HB. Patient and hospital characteristics associated with inpatient severe sepsis mortality in California, 2005-2010. Crit Care Med. 2012;40(11):2960-2966.

3. Melamed A, Sorvillo FJ. The burden of sepsis-associated mortality in the United States from 1999 to 2005: an analysis of multiple-cause-of-death data. Crit Care. 2009;13(1):R28.

4. Jha AK, Shlipak MG, Hosmer W, Frances CD, Browner WS. Racial differences in mortality among men hospitalized in the Veterans Affairs health care system. JAMA. 2001;285(3):297-303.

5. Sarrazin MV, Cannon KT, Rosenthal GE, Kaldjian LC. Racial differences in mortality among veterans hospitalized for exacerbation of chronic obstructive pulmonary disease. J Natl Med Assoc. 2009;101(7):656-662. 
6. Mayr FB, Yende S, Linde-Zwirble WT, Peck-Palmer OM, Barnato AE, Weissfeld LA, Angus DC. Infection rate and acute organ dysfunction risk as explanations for racial differences in severe sepsis. JAMA. 2010;303(24):24952503.

7. O'Brien JM, Jr., Lu B, Ali NA, Levine DA, Aberegg SK, Lemeshow S. Insurance type and sepsis-associated hospitalizations and sepsis-associated mortality among US adults: a retrospective cohort study. Crit Care. 2011;15(3):R130.
8. Harris PA, Taylor R, Thielke R, Payne J, Gonzalez N, Conde JG. Research electronic data capture (REDCap)a metadata-driven methodology and workflow process for providing translational research informatics support. J Biomed Inform. 2009;42(2):377-381.

9. Harris PA, Taylor R, Minor BL, Elliott V, Fernandez M, O'Neal L, McLeod L, et al. The REDCap consortium: Building an international community of software platform partners. J Biomed Inform. 2019;95:103208. 